\title{
fMRI assessment of upper extremity related brain activation with an MRI-compatible manipulandum
}

\author{
Journal Article \\ Author(s): \\ Yu, Ningbo; Estévez, Natalia; Hepp-Reymond, Marie-Claude; Kollias, Spyros S.; Riener, Robert \\ Publication date: \\ 2011-05 \\ Permanent link: \\ https://doi.org/10.3929/ethz-b-000022291
}

Rights / license:

In Copyright - Non-Commercial Use Permitted

Originally published in:

International Journal of Computer Assisted Radiology and Surgery 6(3), https://doi.org/10.1007/s11548-010-0525-5 


\title{
fMRI assessment of upper extremity related brain activation with an MRI-compatible manipulandum
}

\author{
Ningbo Yu • Natalia Estévez • \\ Marie-Claude Hepp-Reymond • \\ Spyros S. Kollias - Robert Riener
}

Received: 11 January 2010 / Accepted: 15 July 2010 / Published online: 10 August 2010

(C) CARS 2010

\begin{abstract}
Purpose Longitudinal studies to evaluate the effect of rehabilitative therapies require an objective, reproducible and quantitative means for testing function in vivo. An fMRI assessment tool for upper extremity related brain activation using an MRI-compatible manipulandum was developed and tested for use in neurorehabilitation research.

Methods Fifteen healthy, right-handed subjects participated in two fMRI sessions, which were three to four weeks apart. A block design paradigm, composed of three conditions of subject-passive movement, subject-active movement and rest, was employed for the fMRI recordings. During the rest
\end{abstract}

This work was supported in part by the Swiss National Science Foundation NCCR on Neural Plasticity and Repair, project P8 Rehabilitation Technology Matrix as well as ETH Research Grant TH-34 06-3 MR-robotics.

N. Yu and N. Estévez made equal contribution to this work.

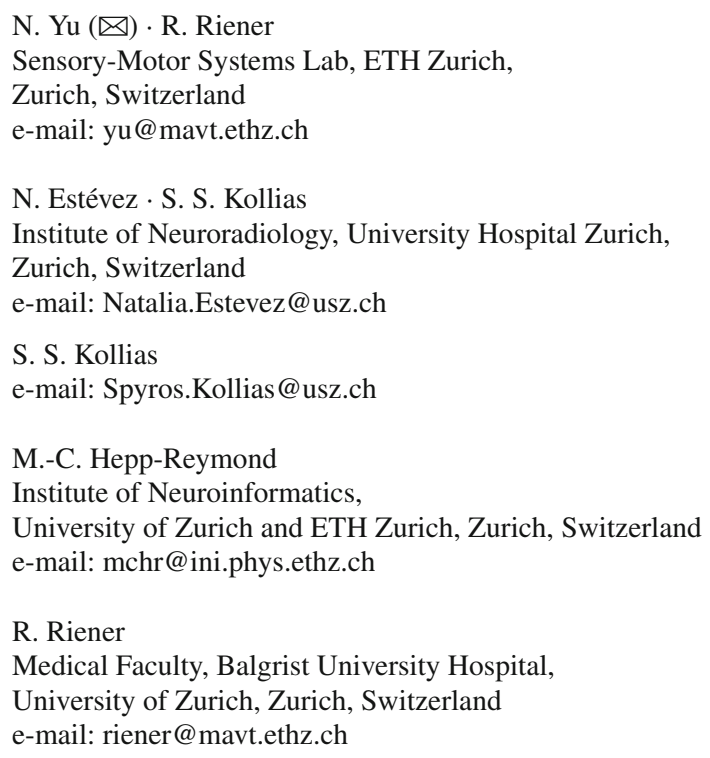

condition, subjects simply held the device handle without applying any force or movement. The same type of auditory and visual instructions were given in all the three conditions, guiding the subjects to perform the motor tasks interactively with the MRI-compatible arm manipulandum. The tasks were controlled across the fMRI sessions. The subjects' brain activation was recorded by fMRI, and their behavioral performance was recorded by the manipulandum. The brain network activated by the subjects' interaction with the manipulandum was identified, and the reproducibility and reliability of the obtained activation were determined.

Results All subjects completed the trial protocol. Two subjects were excluded from analysis due to head motion artifacts. All passive movements were performed well. Four out of the total 780 active movements were missed by two subjects. Brain activation was found in the contralateral sensorimotor cortex, secondary somatosensory cortex and non-primary motor cortex as well as in subcortical areas in the thalamus, basal ganglia and the cerebellum. These activations were consistent across the two fMRI sessions.

Conclusion The MRI-compatible manipulandum elicited robust and reproducible brain activations in healthy subjects during the subject-active and subject-passive upper extremity motor tasks with a block design paradigm. This system is promising for many applications in neurorehabilitation research and may be useful for longitudinal studies.

Keywords Brain activation - fMRI - MRI-compatible manipulandum $\cdot$ Neurorehabilitation

\section{Introduction}

Functional Magnetic Resonance Imaging (fMRI) is an established clinical diagnostic method as well as an indispensable tool in clinical research. It allows brain function to be 
measured in a non-invasive manner and therefore allows repeated measurements over time in order to address questions related to brain reorganization after central and peripheral damage or plasticity following training. To ensure that the participants perform a designed motor task in the same manner, the performance of the task must be adequately controlled and monitored. Furthermore, task control and monitoring can be of great importance for studying effects of rehabilitative therapies.

Tasks commonly used to study brain function - for example wrist flexion-extension, finger tapping or arm flexionextension [1-3] — do not allow optimally controlled studies in patients, due to the difficulty of ensuring consistency in the repetition of each task in impaired subjects whose motor functions are changing over time or across subjects (inter- and intra-subject variability) $[4,5]$. Individual variability across fMRI sessions may confound brain activation changes following a rehabilitative intervention. Therefore, a reduction in the number of uncontrolled variables is essential for the accurate determination of functional brain maps in humans and for the understanding of rehabilitation processes in patients.

MRI-compatible robotic devices can overcome the aforementioned limitations by providing control and monitoring of motor tasks [5-9]. They are able to guide subjects to passively perform well-controlled and reproducible sensorimotor tasks $[5,6]$. Besides, they can work as a haptic interface under closed-loop control so that subjects can move the robotic device in an interactive manner, i.e., active movements that depend on effort of the subjects. Furthermore, the movement parameters can be measured and recorded by the robotic system, which will facilitate the fMRI data analysis afterward. All these special features enable MRI-compatible robots as a great tool to improve neurorehabilitation by providing a more controlled method of gaining insight into the brain reorganization mechanism after damage to the central or peripheral nervous systems and to objectively monitor the effect of therapy at brain level.

This study utilized an established MRI-compatible arm manipulandum, which is safe to be placed into the MRI environment, works compatibly with fMRI and allows extension and flexion of the elbow joint. The main goals of this study were to (1) define the brain network activated by the subjects' interaction with this MRI-compatible arm manipulandum while performing voluntary (active subject) and guided (passive subject guided by the manipulandum) movements, (2) examine the reproducibility and reliability of activation obtained in healthy subjects by fMRI measurements using this device, and eventually (3) determine whether this device is suitable for use in future longitudinal studies to evaluate the effect of various rehabilitative therapies. The longitudinal studies will allow us to correlate functional recovery with specific brain activation patterns, which promises important insights into the ongoing recovery process.

\section{Methods}

Subjects and the MRI setup

The study was approved by the local ethics committee. Fifteen healthy subjects (seven female, eight male, age range: 20-31) were recruited to join this study. All participants gave their written consent for their participation in the study. None of the subjects had any history of neurological or psychiatric disorder. According to the Edinburgh-handedness inventory, all subjects showed right-hand dominance.

The study was carried out in the MR-Center of University of Zurich and ETH Zurich, on a Philips Achieva 1.5 T MR system equipped with an 8 channel SENSETM head coil. The functional acquisitions used a $\mathrm{T}_{2}^{*}$ weighted, single-shot, field echo, EPI sequence of the whole brain $(\mathrm{TR}=3 \mathrm{~s}, \mathrm{TE}=50$ $\mathrm{ms}$, flip angle $=82^{\circ}, \mathrm{FOV}=220 \mathrm{~mm} \times 220 \mathrm{~mm}$, acquisition matrix $=128 \times 128$, in-plane resolution $=1.7 \mathrm{~mm} \times 1.7 \mathrm{~mm}$, slice thickness $=4 \mathrm{~mm}$, SENSE factor 1.6). Additionally, anatomical images of the whole brain were acquired using a $3 \mathrm{D}, \mathrm{T}_{1}$-weighted, field echo sequence $(\mathrm{TR}=20 \mathrm{~ms}, \mathrm{TE}=$ $4.6 \mathrm{~ms}$, flip angle $=20^{\circ}$, in-plane resolution $=0.9 \mathrm{~mm} \times$ $0.9 \mathrm{~mm}$, slice thickness $=0.75 \mathrm{~mm}, 210$ slices $)$.

\section{The MRI-Compatible manipulandum}

The manipulandum (Fig. 1) is safe to be placed inside the scanner room for Magnetic Resonance Imaging (MRI) and is able to work together with MRI and functional MRI (fMRI) procedures [6].

During this investigation, the MRI-compatible manipulandum including the actuator and sensors was placed inside the MRI scanner room (Fig. 1, top). Digital components including the control unit, electric motors powering the hydraulic actuator, electric circuit, and other parts of the system, were placed outside the scanner room. Control valves and pressure sensors were placed at the corner of the scanner room, far away from the end-effector which was located inside the scanner bore. Optical fibres, cables, and hoses transmitted signals and fluid power through the shielding wall of the MRI scanner room.

The position, height and orientation of the manipulandum can be adjusted to to fit the size and movement preference of subjects (Fig. 1, bottom). These parameters constrain the movement of the manipulandum. The manipulandum interacts with human subjects with a handle, which was attached to and driven by a hydraulic cylinder. The cylinder was specially made of bronze and aluminum so that it can be used inside the MRI room. This hydraulic cylinder enables the handle a linear movement range of $25 \mathrm{~cm}$, velocity range of $20 \mathrm{~cm} / \mathrm{s}$ and force range of up to $300 \mathrm{~N}$. A selfdesigned and self-manufactured optical force sensor, which is adapted from [10] was installed between the handle and the 

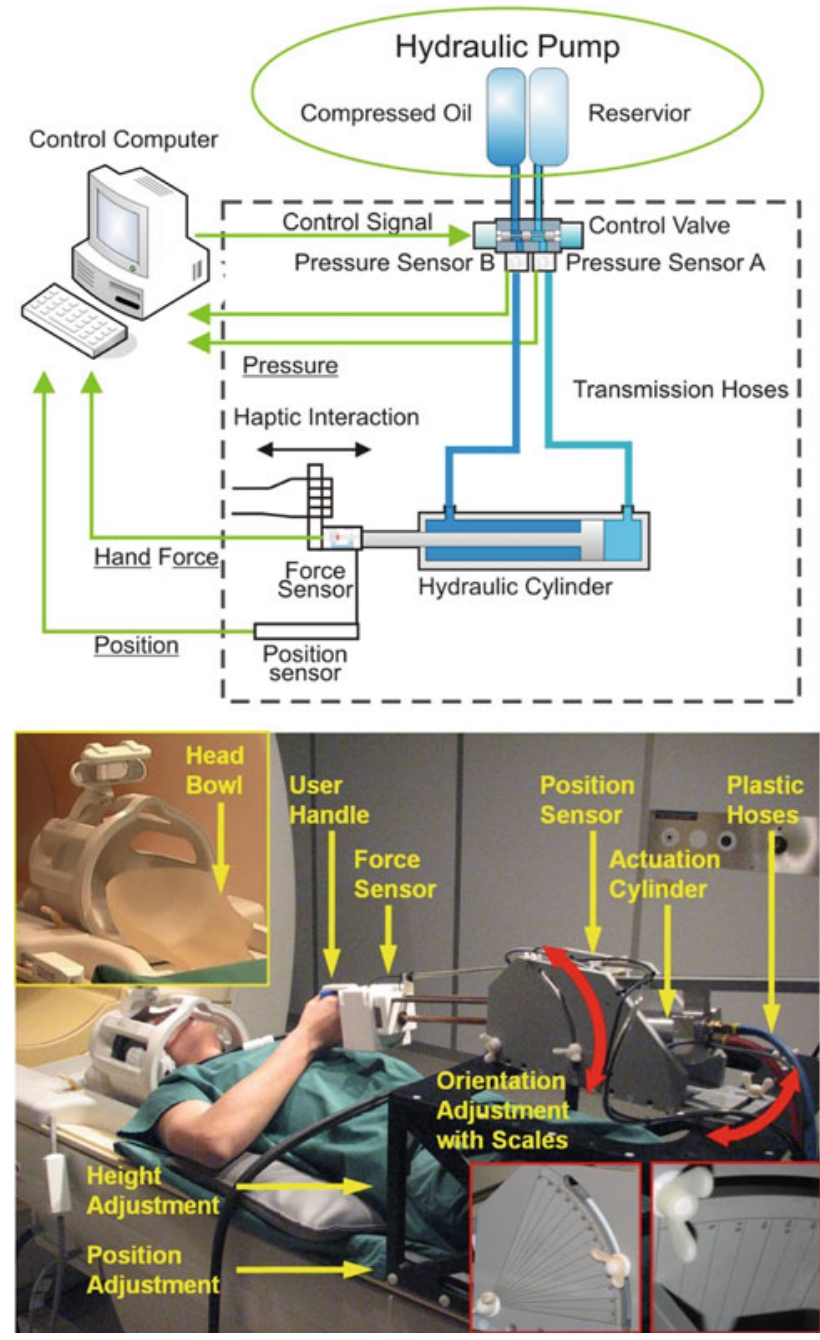

Fig. 1 Top schematic plot of the MRI-compatible manipulandum system. Bottom a subject with the MRI-compatible manipulandum in the MRI scanner room

cylinder, measuring the push and pull force from the subject's arm to the cylinder. This force sensor can measure up to $120 \mathrm{~N}$ in both directions. An optical encoder, LIDA 279 by Heidenhain, measures the position of the handle. A special potential meter, MTP-L 22 by Resenso, was used as a redundant position sensor. For other components, PVC and PET were carefully selected as the main construction materials [6].

In order to reduce head motion artifacts during the data acquisition, we used a self-made head support, which covered the superior and partially the lateral parts of the subjects head (Fig. 1, bottom). This limited the range of head motion, especially in the spinal direction. Furthermore, foam pillows were used to additionally restrict the motion in the left-right direction.

Under position control, the manipulandum can guide a subject to perform linear smooth movements. Under admittance control, the manipulandum is able to interact with subjects in various resistance laws, such as the spring law (resistance proportional to displacement), the viscous law (resistance proportional to speed), combination of the two, or some special-purpose resistance laws [6]. Specially, the manipulandum is able to receive external commands via an RS232 cable and then switch freely between the position control mode and the admittance control mode. Therefore, the manipulandum is able to

- guide the subject's arm to perform pre-defined linear movements;

- interact with the subject's arm with various kinds of resistance;

- receive external commands and produce the corresponding active or passive movements;

- record the position information of the movement;

- record pull or push force from the arm to the cylinder during the movement.

Phantom test with the manipulandum

The manipulandum was able to work safely and properly inside the MRI scanner room. Before the functional study with human subjects, a phantom test was performed to examine whether the manipulandum disturbed the MRI system. The experiment covered the following conditions:

(1) phantom only, in which the manipulandum was not in the scanner room;

(2) device silent, in which the manipulandum was placed at its desired working location in the scanner room, but had no connection going out of the scanner room;

(3) device powered on, in which the manipulandum was placed at its desired working location in the scanner room, with all transmission lines connected and the whole system powered on, but not performing any task;

(4) device functioning, in which the manipulandum performed the passive movements at its desired working location.

The imaged phantom was a bottle of mineral oil. In each of these experimental conditions, 20 fMRI scans were acquired for the phantom. The slice closest to the manipulandum would be most vulnerable to possible disturbances from the device and therefore was taken as the benchmark for evaluation of possible image artifacts.

Two parameters of interest were to be inspected: the signal-to-noise ratio (SNR) and the temporal signal-to-noise ratio (tSNR). The SNR was calculated as:

$\mathrm{SNR}=\frac{\text { mean signal in image ROI }}{\text { standard deviation in image ROI }}$ 


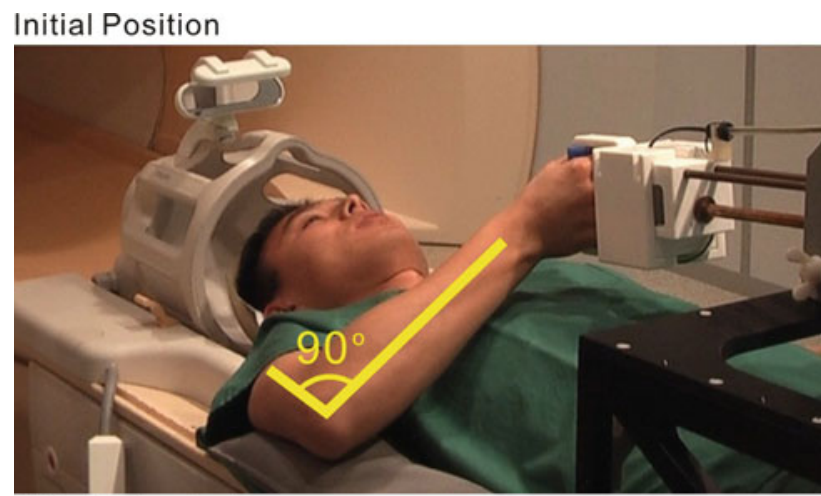

Wrist Extended

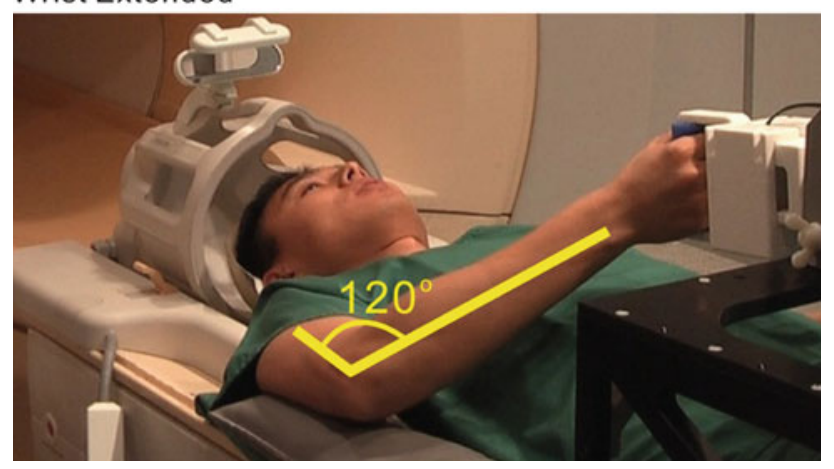

Fig. 2 The arm extends about $30^{\circ}$ when the handle linearly moves about $20 \mathrm{~cm}$

The tSNR was calculated as

$\mathrm{tSNR}=\frac{\text { mean of voxel time series }}{\text { standard deviation of voxel time series }}$

The signal, noise and SNR values were calculated for all the 20 images at the selected slice, and then averaged. SNR and tSNR will be given in $\mathrm{dB}^{1}$.

fMRI Motor tasks and experimental paradigm

For fMRI scans, the participants were asked to lie on the MRI bench and the fixation frame was positioned above the subjects' thighs. Afterward, the participants were asked to flex the right elbow to reach the handle. The position, height and orientation of the manipulandum were adjusted to ensure that subjects reached the handle and performed the functional tasks in a comfortable way, while the upper arm remained close to the body without causing shoulder and head motion (Fig. 2). Additionally, the elbow was supported by a cushion for better comfort and stabilization of the upper arm.

To investigate the subjects' motor interactions with the MRI-compatible manipulandum, the experiment consisted of three conditions: rest, subject-passive movement and subject-active movement. In the passive movement condition,

${ }^{1}$ Decibel: (number in $\left.\mathrm{dB}\right)=20 \log _{10}$ (number in decimal).

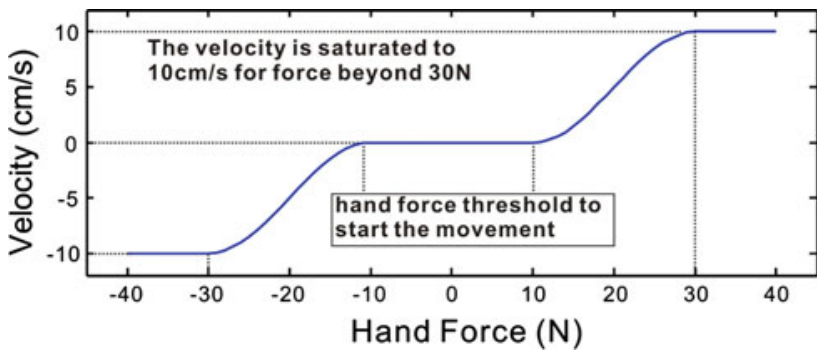

Fig. 3 The force-velocity profile employed in the active mode of the study

subjects were required to hold the device's handle and follow its movement without applying any force to it. The speed was constantly $7.2 \mathrm{~cm} / \mathrm{s}$. In the active movement condition, by contrast, subjects had to push and pull actively to produce the movement. The force-velocity profile adopted for this mode was shown in Fig. 3. The movement could only be initiated after the force reached a certain threshold. Above this threshold, an inverse viscous law was applied in the sense that the more force the subject applied, the faster the arm moved. The maximal speed was saturated to $10 \mathrm{~cm} / \mathrm{s}$ when the force reached $30 \mathrm{~N}$ or beyond. The low speed and smooth movements were used for both active and passive movements in order to avoid head motion, and thus, potential artifacts to brain images $[6,11]$.

The range of motion for the handle was about $16-20 \mathrm{~cm}$ depending on the postural and kinematic (movement direction/orientation) preferences as well as the size of individual subjects. For each subject, the range of motion and linear movement trajectory remained the same for all passive and active movements. The speed was smoothly reduced to zero at the two endpoints.

The force thresholds were normalized to the capability of the subjects, defined as $20 \%$ of their maximal voluntary push force. This force was assessed with the MRI-compatible manipulandum for each subject before either fMRI scanning. Participants were instructed to push the fixed handle of the manipulandum three times with their maximal voluntary force without moving head and body, and the mean force value was taken.

A block design (Fig. 4) with 29 s periods of rest alternating with $29 \mathrm{~s}$ periods for each movement condition was used. The two movement conditions were presented in a pseudorandom order (ABBAAB, A for passive and $\mathrm{B}$ for active) and repeated ten times. Each active or passive movement block was composed of three repetitions of the push and pull movement with a small pause between the repetitions. Hence, there were a total of 30 active and passive movements in the whole run, which lasted about 20 min. Passive and active movements were visually and acoustically guided to ensure the active movements had approximately the same duration as the passive ones. The visual instruction was displayed on a 


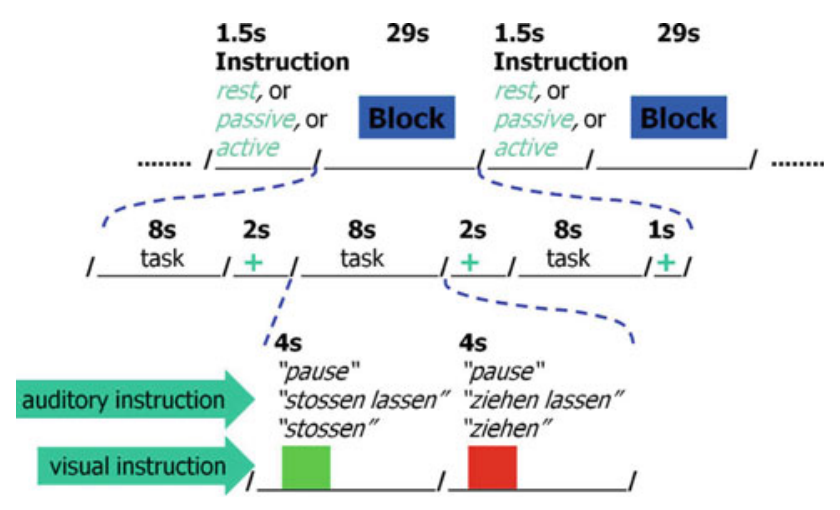

Fig. 4 The block design paradigm

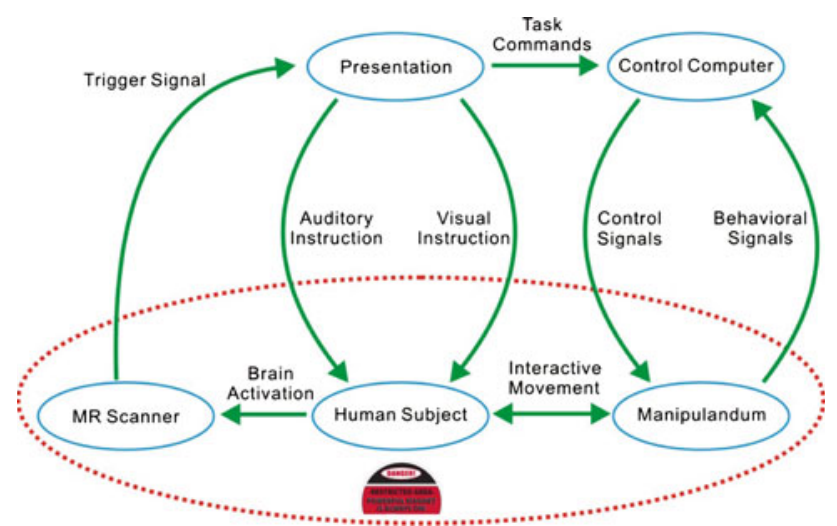

Fig. 5 Illustration of the study: human subject, involved components and their interactions

screen and consisted of a green and a red square. Each square was presented for $4 \mathrm{~s}$ and the green one was presented always first. During the active condition, participants were instructed to push the device when the green square was presented and to pull it when the red one was displayed. The auditory instruction for the active condition consisted of the words "stossen" (German: "to push") and "ziehen" (German: "to pull"), which were synchronized with the green and red squares, respectively. During the rest and passive movement conditions, exactly the same colored squares were presented and the participants were asked to fixate the squares. The auditory instruction for the passive and rest conditions consisted of the words "stossen lassen" (German: "to be pushed") and "ziehen lassen" (German: "to be pulled") for the passive condition and "Pause" (German: "pause") for the rest condition. The experimental paradigm was implemented by the program Presentation (http://www.neurobs.com/). It received trigger signals from the MRI system, provided the visual and auditory instructions to the subjects, and sent control commands to the manipulandum (Fig. 5). With Presentation, the brain activation data and the behavioral data were synchronized.

The subjects were trained to practice the tasks prior to the scanning procedures outside of the scanner bore so that the designed tasks were executed properly. The fMRI session was repeated for all subjects three to four weeks after the first fMRI session to examine the repeatability and robustness of the brain activation elicited by the interested arm tasks.

Data analysis

Parameters of interest for assessing the motor performance in each block were the number of movements, the range of motion for each movement, and the force in each movement. These parameters were examined to check whether the functional tasks were executed in the desired way and were compared across active and passive conditions as well as the two fMRI sessions.

Image processing and analysis were performed using SPM8 (Welcome Department of Cognitive Neurology, London, http://fil.ion.ucl.ac.uk/spm) implemented in MATLAB 7.6 (Mathworks Inc., Natick, MA, USA). Data pre-processing was carried out for each subject prior to the computation of group analysis. Images were motion corrected by means of 7th Degree B-Spline interpolation (6-parameter spatial transformation). The movement parameters obtained during this procedure were used to determine the extent of movements. The data of participants that did not exceed a value of about half of the voxel size was included in the analysis. Functional images were normalized into standard space using the Montreal Neurological Institute template (MNI). Spatial smoothing was performed by applying a Gaussian filter of $6 \mathrm{~mm}$ full-width at half-maximum (FWHM), to reduce the noise and enhance the signal. Additionally, a high-pass filter was applied to remove slow temporal drifts with a period longer than $256 \mathrm{~s}$.

The statistical analysis was performed at two levels. At the first level, the experimental conditions were modeled by the general linear model (GLM) using a canonical hemodynamic response function. To further correct residual movement artifacts that were not removed by the previously mentioned motion correction procedure, the translation parameters obtained from this procedure were included in the design matrix of the model. Model estimation was performed on a subject-by-subject basis for each session separately in order to identify the general networks involved in the subject-active and subject-passive tasks by contrasting the induced brain activation with that in the rest condition. At the second level, group analysis was performed according to the random effects analysis using the single subject contrast images obtained in the first step as input. One-sample $t$-tests were generated for each movement condition versus the rest condition and also for the comparison of two movement conditions, for each session separately. The significance level for the resulting statistical maps was set at $P<0.0001$ (extent threshold $k=10$ ). To assess reproducibility and robustness of the brain activation elicited by the 
Table 1 Phantom test: signal, noise, and signal-to-noise-ratio (SNR)

\begin{tabular}{llll}
\hline Condition & SNR $(\mathrm{dB})$ & Signal & Noise \\
\hline Phantom only & $38(0.8)$ & $1801(2.8)$ & $21.6(2.1)$ \\
Device silent & $38(0.8)$ & $1775(4.0)$ & $22.9(2.3)$ \\
Device poweredON & $38(0.9)$ & $1769(4.0)$ & $22.4(2.3)$ \\
Device functioning & $37(0.9)$ & $1775(4.3)$ & $24.8(2.6)$ \\
\hline
\end{tabular}

Values are given as: mean (standard deviation)

Table 2 Phantom test: temporal signal, temporal noise, and temporal signal-to-noise-ratio (tSNR)

\begin{tabular}{llll}
\hline Condition & tSNR(dB) & Signal & Noise \\
\hline Phantom only & $44(1.7)$ & $1801(18.7)$ & $11.5(2.2)$ \\
Device silent & $43(1.5)$ & $1775(19.6)$ & $12.7(2.1)$ \\
Device poweredON & $42(1.3)$ & $1769(18.4)$ & $13.8(2.0)$ \\
Device functioning & $40(1.7)$ & $1775(17.9)$ & $17.9(3.2)$ \\
\hline
\end{tabular}

Values are given as: mean (standard deviation)

functional tasks, two-sample $t$-tests were generated for each contrast in each session, with a threshold at $P<0.0001$ (extent threshold $k=10$ ). Family-wise correction was not applied to the statistical tests, and this threshold was chosen because it is less conservative. Therefore, it could be more informative and show better activations in the expected network.

\section{Results}

\section{Phantom test}

The SNR and tSNR values of the nearest slice to the manipulandum were shown in Tables 1 and 2 .

It could be observed that good signal, high SNR and tSNR were obtained in all phantom experiments. Neither the introduction of the manipulandum into the MRI environment nor its functioning brought notable spatial or temporal disturbances to the fMRI procedures. Besides, visual inspection did not find significant differences among images obtained in different conditions. Therefore, it has been demonstrated that the manipulandum did not interfere with fMRI procedures.

\section{Behavioral performance}

All the subjects accomplished the two fMRI sessions and no subject reported any discomfort. Two subjects (one female, one male) were excluded from the analysis due to significant movement artifacts.

All passive movements were performed as designed in both fMRI sessions. During the active movement condition, a total of 390 movements were designed for all the thirteen

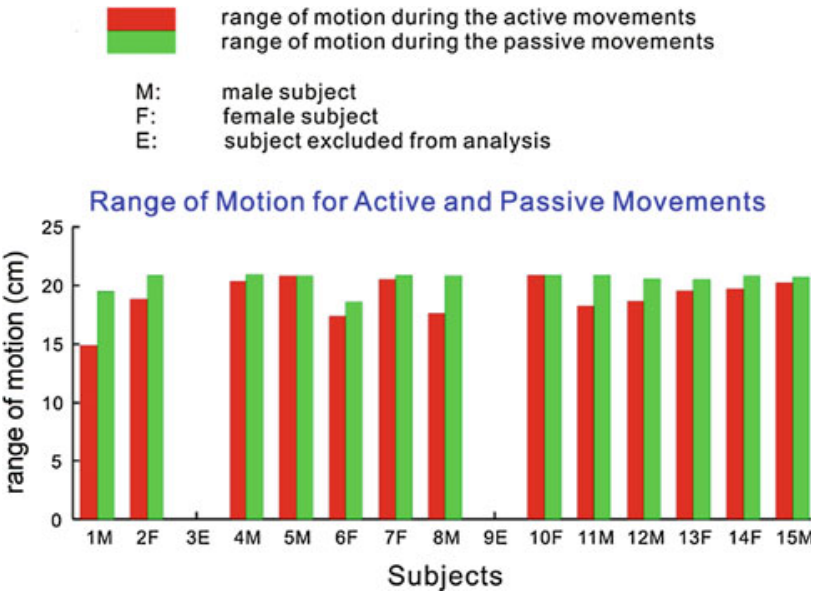

Fig. 6 Range of motion during active and passive movements

subjects in each fMRI session. All active movements were performed in the first fMRI session, and four active movements were missed by two subjects in the second fMRI session. In general, the behavioral performance in the two fMRI sessions was quite similar, and no significant difference was observed.

The range of motion during passive movements varied from 16 to $20 \mathrm{~cm}$ between subjects depending on their size which caused some adjustments of the manipulandum. In the active condition, the range of motion depended on the voluntary effort of the subjects and was reduced compared to the passive condition (Fig. 6).

When comparing the second with the first session for active movements, the average range of motion increased from 16.5 to $19.0 \mathrm{~cm}$, although the average force decreased from 20.1 to $17.1 \mathrm{~N}$. This can be partially explained by the fact that the average measured maximal force decreased from 54.6 to $46.5 \mathrm{~N}$ and, therefore, the force threshold of movements decreased from 10.9 to $9.3 \mathrm{~N}$. The average force during passive movements increased from 0.2 to $0.6 \mathrm{~N}$ in the second measurement compared to the first measurement (Fig. 7).

\section{Brain activation}

When contrasting active movement condition versus rest in the first fMRI session, brain activation was detected in the contralateral sensorimotor cortex (M1/S1), and bilaterally in the secondary somatosensory cortex (S2), in the supplementary motor area (SMA), cingulate motor areas (CMA), the contralateral dorsal premotor cortex $(\mathrm{PMd})$ and in the insula, (one sample $t$-test, $P<0.0001$, extent threshold ${ }^{2} k=$ 10). Additionally activation was found in the ipsilateral cerebellum, and bilaterally in the posterior cerebellum (CB), the thalamus and basal ganglia. During the second fMRI

\footnotetext{
${ }^{2}$ Extent threshold: the minimum cluster threshold.
} 


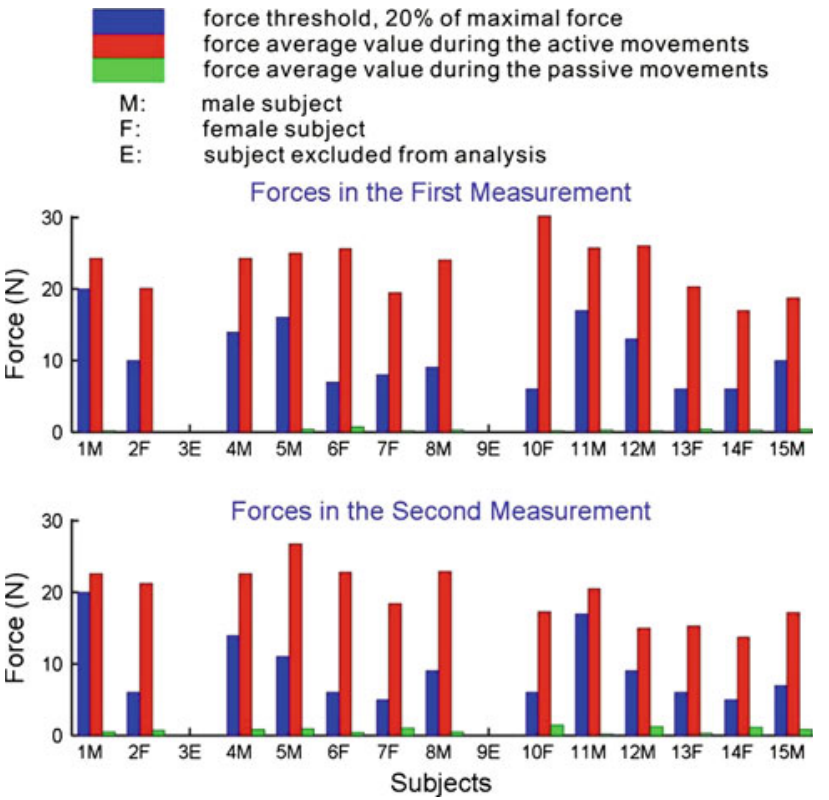

Fig. 7 The measured forces during active and passive movements in the two measurements

session, the same network was significantly activated, except for the posterior CB (Fig. 8).

When contrasting the passive movement condition against rest, activation was found in the same brain regions during both sessions, but not in PMd and insula and only in the ipsilateral cerebellum (one sample $t$-test, $P<0.0001$, extent threshold $k=10$ ) (Fig. 9). One-sample $t$-test analysis showed that activation was stronger in all regions of the aforementioned network during active when compared to passive movements $(P<0.0001$, extent threshold $k=10)$. In the second session, the contrast between active and passive movements showed again significantly more activation during active movements, except in contralateral S2.

Although activation seemed to be stronger in all mentioned brain regions for the three contrasts during the first session compared to the second one, two-sample $t$-test analysis did not reveal significant differences between the two sessions in all these contrasts $(P<0.0001$, extent threshold $k=10)$.

Additionally, activation was found in primary and secondary visual areas especially in the right hemisphere, although the same visual instruction was shown in all conditions and consequently the occipital activation should have been removed. This activation was stronger during the performance of active movements compared to passive movements. A possible explanation for this activation may be that participants saw part of the manipulandum while they had to move the handle actively or when the handle was moving by its own.

\section{Discussion and conclusion}

The subjects' interaction with the new MRI-compatible manipulandum elicited activation in a brain network that
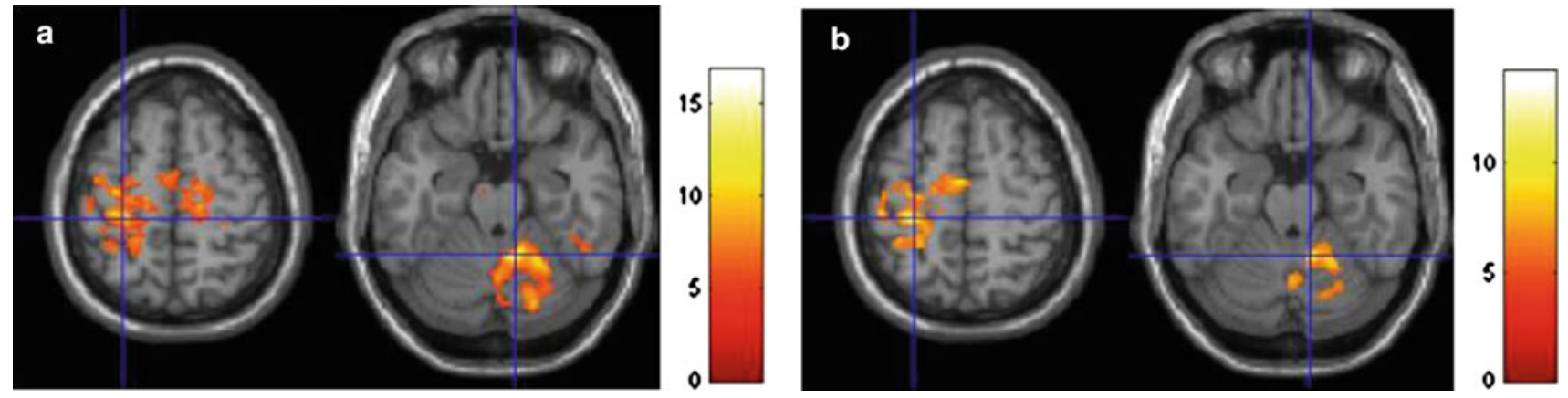

Fig. 8 Activation for the contrast active movement versus rest $\mathbf{a}$ in the first session and $\mathbf{b}$ in the second session
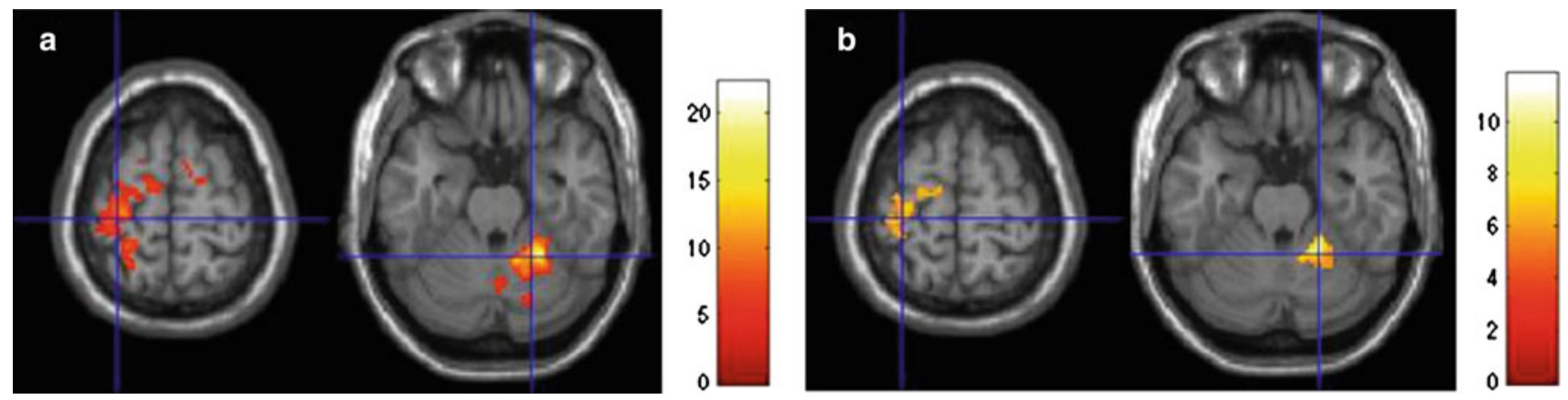

Fig. 9 Activation for the contrast passive movement versus rest $\mathbf{a}$ in the first session and $\mathbf{b}$ in the second session 
included mainly the primary sensorimotor cortex, secondary somatosensory and medial and lateral premotor areas, as well as subcortical regions during the performance of passive and active movements. These findings are largely consistent with an earlier investigation on passive and active elbow movements [12]. In addition, activation in these areas was stronger when participants were voluntarily moving the handle than when they were guided by the manipulandum. This stronger activation may be explained by the fact that participants were applying voluntary force during the active condition but not during the passive one. Several studies already showed that increased force leads to stronger activation in the sensorimotor cortex [13-15].

When the two sessions were compared, slight changes in the measured parameters were observed. In the second session, the range of motion during active movements was bigger and the voluntary force during active movements was lower. This is probably due to the fact that the maximal voluntary force was lower on average in the second session, leading to a lower force threshold. The two fMRI sessions showed no statistically significant differences in the brain activation. Previous findings in literature on reproducibility of brain activation using functional imaging techniques are controversial. In some studies, repetition of specific tasks induced changes in brain activation [16,17], while other studies reported robust activation across sessions $[18,19]$. A lack of reproducibility can be accounted by multiple factors such as familiarity to the MRI experiment and environment. Less attention, stress and memory effects may also reduce brain activation when participants become familiar with the procedure. For instance, Loubinoux and colleagues [16] suggested that a long-term memory representation of the sensorimotor task can be implemented into the motor system along the sessions, leading to differences in cortical activation. Further, differences in task performance may influence the recorded brain activation and lead to inter-session variances. While some confounding variables, such as familiarity, cannot be controlled precisely, differences in task performance can be monitored by MRI-compatible devices, which can help to interpret differences in brain activation between sessions. Furthermore, MRI-compatible devices allow well-controlled and reproducible tasks and thus allow comparable sessions. Previous studies that used standardized movements reported high consistency of brain activation, suggesting that the performance of controlled movements can improve the reproducibility of brain activation $[18,19]$. In our investigation, we used a novel MRI-compatible manipulandum that allows adjustable, well-controlled and reproducible passive movements across fMRI sessions and subjects, interactive movements with various kinds of resistance, free switch between the active and passive movements under external control, and recording of the behavioral information such as position and force. The strong controlled settings enabled the re-occurrence of the same active and passive movements several weeks after, without inducing significant changes in brain activation.

Our study is promising for long-term studies in clinical settings with the application of MRI-compatible devices in the MRI environment to perform various functionally meaningful tasks. This suggests that our device can be used as an MRI-compatible tool to explore brain reorganization following injury and to evaluate rehabilitative interventions in patients suffering from damage to the central or peripheral nervous systems.

Acknowledgment The authors would like to thank Prof. Roger Gassert, Dr. Peter Wolf, Dr. Michael Brügger, Christoph Hollnagel, Dr. Birgit Keisker, Michael Villiger and Dr. Martin Meyer for their helpful instructions and discussions. The authors would also like to thank Dr. Roger Lüchinger for his technical support in the MR center. Special thanks go to all the participants of the study.

\section{References}

1. Cramer SC, Benson RR, Himes DM, Burra VC, Janowsky JS, Weinand ME, Brown JA, Lutsep HL (2005) Use of functional MRI to guide decisions in a clinical stroke trial. Stroke 36(5):e50-e52

2. Luft AR, McCombe-Waller S, Whitall J, Forrester LW, Macko R, Sorkin JD, Schulz JB, Goldberg AP, Hanley DF (2004) Repetitive bilateral arm training and motor cortex activation in chronic stroke: a randomized controlled trial. JAMA 292(15): $1853-1861$

3. Pariente J, Loubinoux I, Carel C, Albucher JF, Leger A, Manelfe C, Rascol O, Chollet F (2001) Fluoxetine modulates motor performance and cerebral activation of patients recovering from stroke. Ann Neurol 50(6):718-729

4. Hidler J, Hodics T, Xu B, Dobkin B, Cohen LG (2006) MR compatible force sensing system for real-time monitoring of wrist moments during fMRI testing. J Neurosci Methods 155(2):300307

5. Tsekos NV, Khanicheh A, Christoforou E, Mavroidis C (2007) Magnetic resonance-compatible robotic and mechatronics systems for image-guided interventions and rehabilitation: a review study. Annu Rev Biomed Eng 9:351-387

6. Yu N, Hollnagel C, Blickenstorfer A, Kollias SS, Riener R (2008) Comparison of MRI-compatible mechatronic systems with hydrodynamic and pneumatic actuation. IEEE ASME Trans Mechatron 13(3):268-277

7. Diedrichsen J, Shadmehr R (2005) Detecting and adjusting for artifacts in fMRI time series data. Neuroimage 27(3):624-634

8. Gassert R, Moser R, Burdet E, Bleuler H (2006) MRI/fMRIcompatible robotic system with force feedback for interaction with human motion. IEEE ASME Trans Mechatron 11(2):216224

9. Suminski AJ, Zimbelman JL, Scheidt RA (2007) Design and validation of a MR-compatible pneumatic manipulandum. J Neurosci Methods 163(2):255-266

10. Fueglistaller J (2004) Design and contruction of a dynamometer for use in high magnetic fields. Master thesis

11. Yu N, Hollnagel C, Wolf P, Murr W, Blickenstorfer A, Kollias S, Riener R (2009) Tracking and analysis of human head motion during guided fMRI motor tasks. In: The IEEE international conference on rehabilitation robotics (ICORR) Kyoto, Japan 
12. Weiller C, Juptner M, Fellows S, Rijntjes M, Leonhardt G, Kiebel S, Muller S, Diener HC, Thilmann AF (1996) Brain representation of active and passive movements. Neuroimage 4(2):105-110

13. Dai TH, Liu JZ, Sahgal V, Brown RW, Yue GH (2001) Relationship between muscle output and functional MRI-measured brain activation. Exp Brain Res 140(3):290-300

14. Cramer SC, Weisskoff RM, Schaechter JD, Nelles G, Foley M, Finklestein SP, Rosen BR (2002) Motor cortex activation is related to force of squeezing. Hum Brain Mapp 16(4):197-205

15. Keisker B, Hepp-Reymond MC, Blickenstorfer A, Meyer M, Kollias SS (2009) Differential force scaling of fine-graded power grip force in the sensorimotor network. Hum Brain Mapp 30(8): 2453-2465

16. Loubinoux I, Carel C, Alary F, Boulanouar K, Viallard G, Manelfe C, Rascol O, Celsis P, Chollet F (2001) Within-session and between-session reproducibility of cerebral sensorimotor acti- vation: A test-retest effect evidenced with functional magnetic resonance imaging. J Cereb Blood Flow Metab 21(5):592-607

17. McGonigle DJ, Howseman AM, Athwal BS, Friston KJ, Frackowiak RSJ, Holmes AP (2000) Variability in fMRI: an examination of intersession differences. Neuroimage 11(6):708-734

18. Carey LM, Abbott DF, Egan GF, Tochon-Danguy HJ, Donnan GA (2000) The functional neuroanatomy and long-term reproducibility of brain activation associated with a simple finger tapping task in older healthy volunteers: a serial PET study. Neuroimage 11(2):124-144

19. Alkadhi H, Crelier GR, Boendermaker SH, Golay X, HeppReymond MC, Kollias SS (2002) Reproducibility of primary motor cortex somatotopy under controlled conditions. AJNR 23(9):1524-1532 